Supporting Information

\title{
Heterogeneous, 3D Architecturing of 2D Titanium Carbide (MXene) for Microdroplet Manipulation and Voice Recognition
}

Ye Zhang, ${ }^{1}$ Ting-Hsiang Chang, ${ }^{1}$ Lin Jing, ${ }^{1}$ Kerui Li, ${ }^{1}$ Haitao Yang, ${ }^{1}$ Po-Yen Chen ${ }^{1, *}$

${ }^{1}$ Department of Chemical and Biomolecular Engineering, National University of Singapore (NUS), Singapore 117585.

*Corresponding author. Email: checp@,nus.edu.sg

This file includes:

Note S1 and S2

Figure S1 to S19 


\section{Note S1. Mechanical delamination test.}

The mechanical delamination test was conducted by using a Universal Testing System (Instron) at room temperature. The fabricated s-MXene/PS bilayer devices (with and without oxygen plasma pre-treatment) were cut into squares (ca. $0.5 \mathrm{~cm} \times 0.5 \mathrm{~cm}$ ). Afterwards, the squareshaped s-MXene/PS devices were adhered by super glue in between two pieces of plastic strips

(Figure S4a). The loading force and displacement distances were measured during the delamination tests. The adhesion stress was calculated by the loading force divided by the delaminated areas, and the binding energy between s-MXene nanocoating and PS substrate was obtained by integrating the area of the corresponding curves in Figure S4b. All the measurements were repeated at least for three times to extract the average values. 


\section{Note S2.}

The s-MXene sensor with the shortest papillae $(170 \mu \mathrm{m})$ did exhibit the highest sensitivity of $114 \mathrm{kPa}^{-1}$, yet the practical applications of such s-MXene sensor were technically limited. Due to the smaller spacing between two s-MXene electrodes (ca. $150 \mu \mathrm{m}$ ), a mild curvature of sMXene sensor largely affected the base line of current output signals. Therefore, although such s-MXene sensor exhibited a very high sensitivity, its application to human bodies was largely limited. We often encountered unfavorable short or open circuits during the measurements. Therefore, we rather reported the s-MXene sensor with $330 \mu \mathrm{m}$-height papillae array, which demonstrated high sensitivity and stability for practical exploitations. 
(a)

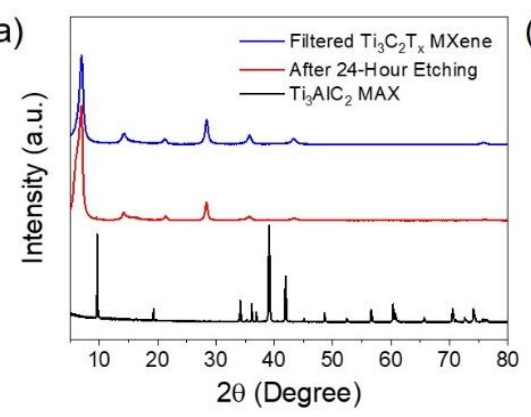

(d)

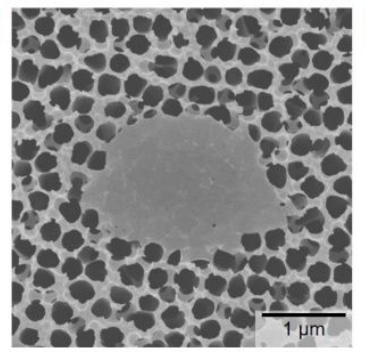

(e) (b)
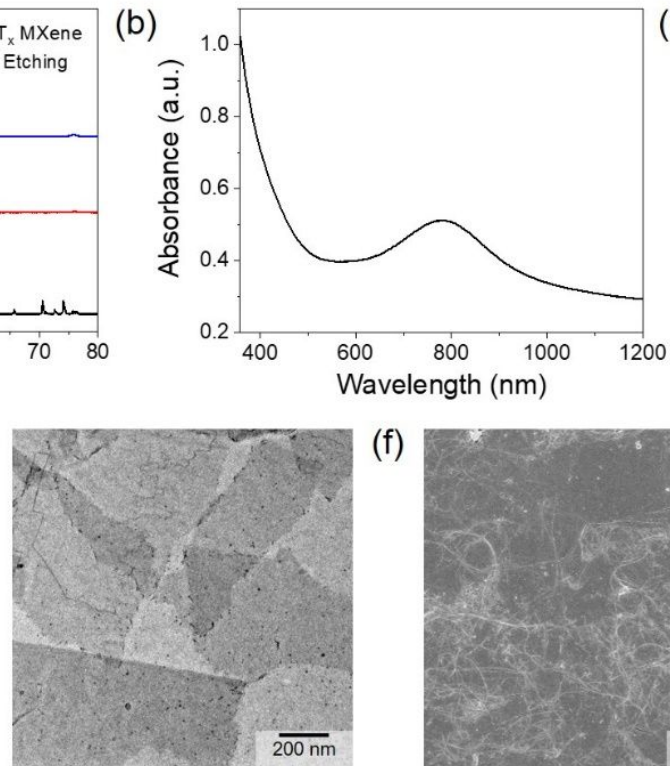

(f)

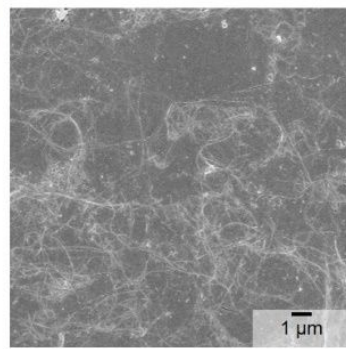

(c)

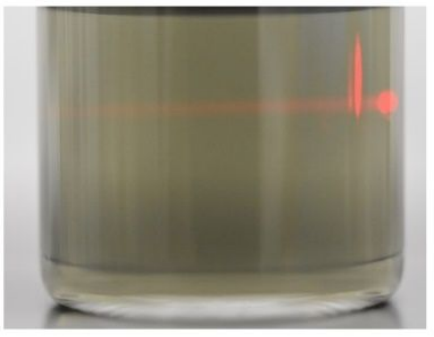

(g)

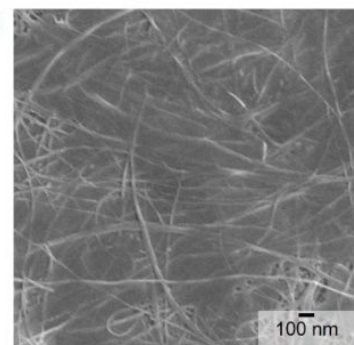

Figure S1. (a) XRD spectra of $\mathrm{Ti}_{3} \mathrm{AlC}_{2}$ MAX particles, MAX etched for 24 hours, and filtered $\mathrm{Ti}_{3} \mathrm{C}_{2} \mathrm{~T}_{\mathrm{x}}$ MXene film. (b) UV-Vis spectrum of dilute MXene dispersion. (c) Tyndall effect of MXene dispersion. (d) SEM and (e) TEM images of as-synthesized MXene nanosheets. (f)(g) Top-down SEM images of the s-MXene film. 
(a)

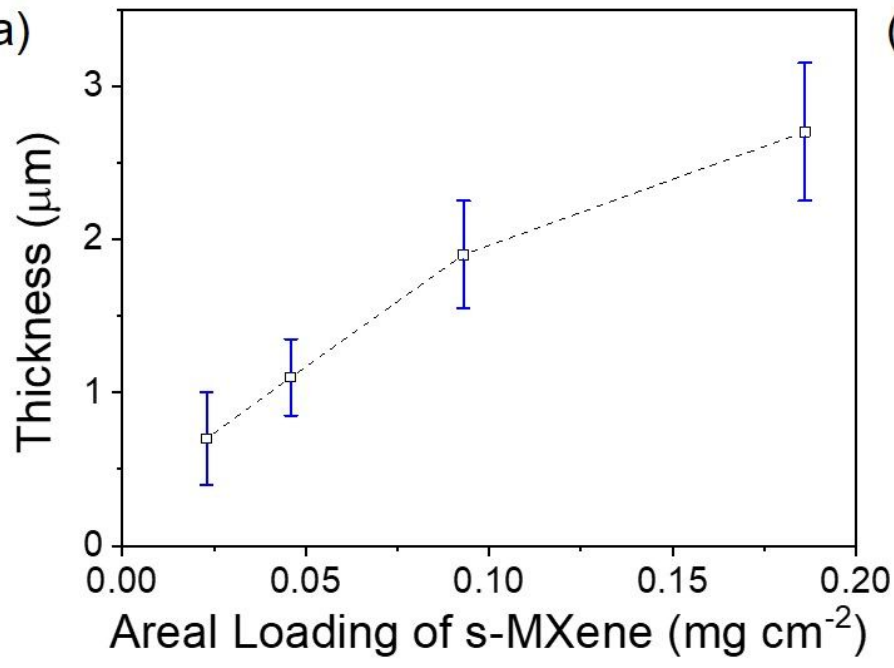

(b)

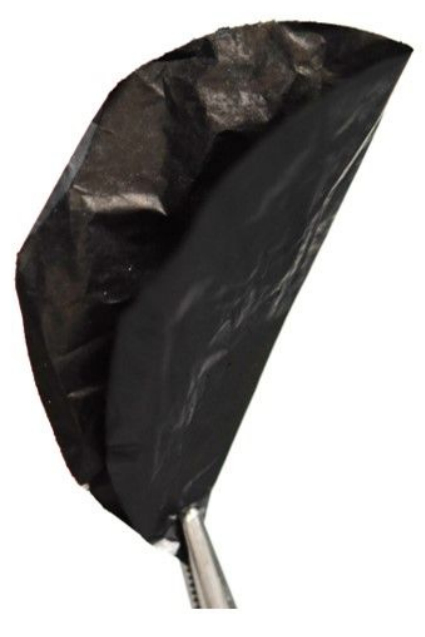

Figure S2. (a) Relation between the thicknesses of s-MXene layers and the areal mass loading of s-MXene filter film. (b) As-filtered, free-standing s-MXene film. 
(a)

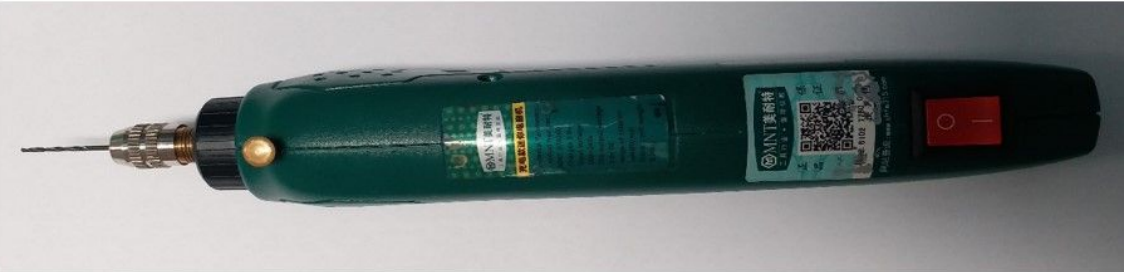

(b)

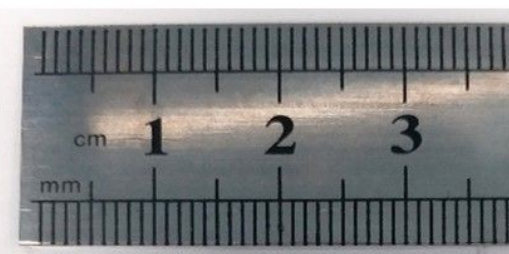

(c)

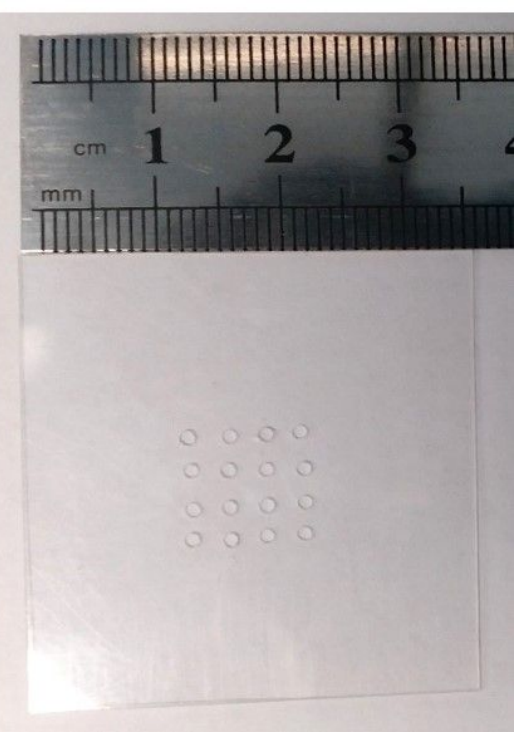

Figure S3. (a) Hand holding drill with micro-bit used in this fabrication. (b) Thermallyresponsive PS shrink film with an array of marked dots. (c) Thermally-responsive PS shrink film with an array of drilled holes (diameter $\sim 1.1 \mathrm{~mm}$ ). 

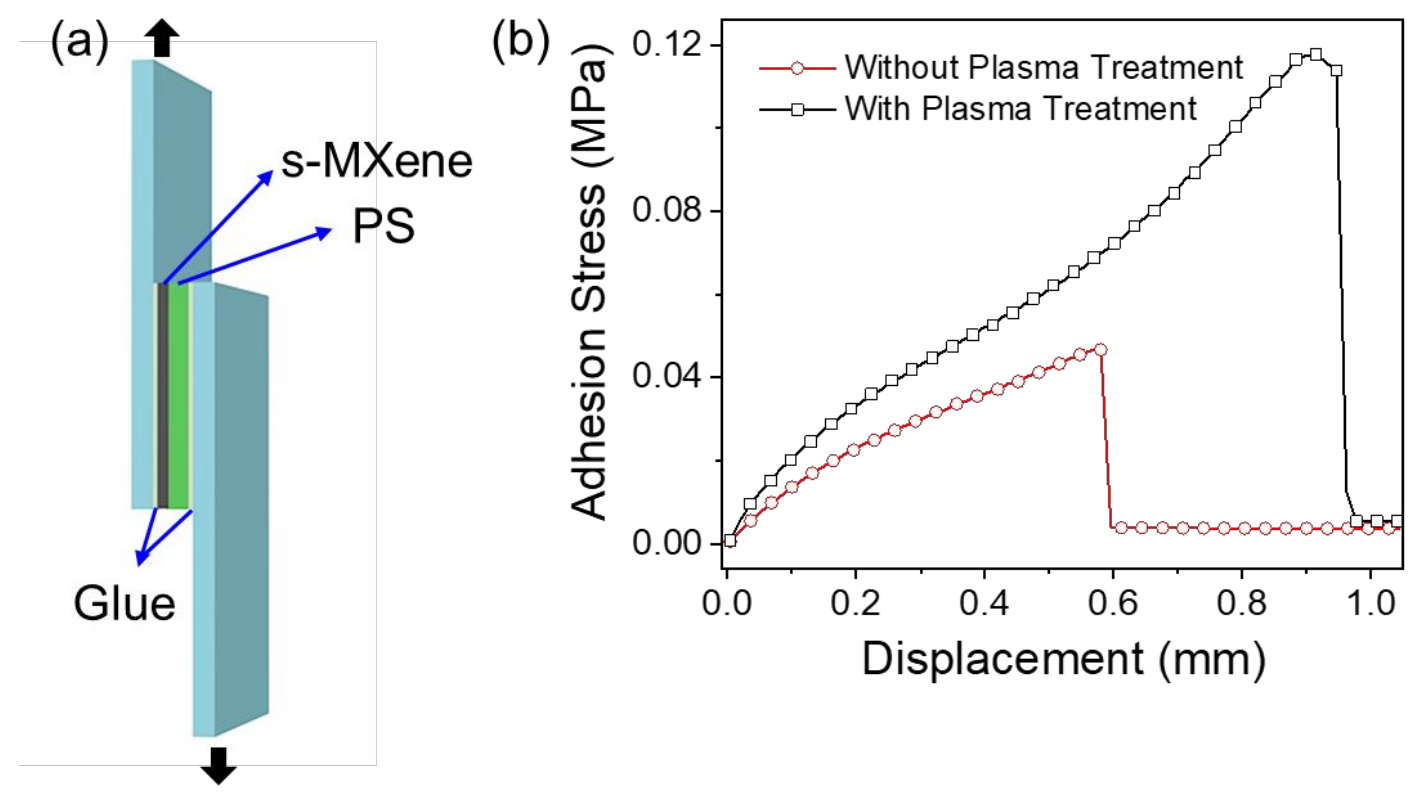

Figure S4. (a) Setup of mechanical delamination test. (b) Testing results of s-MXene/PS devices with and without oxygen plasma pre-treatment.

(a)

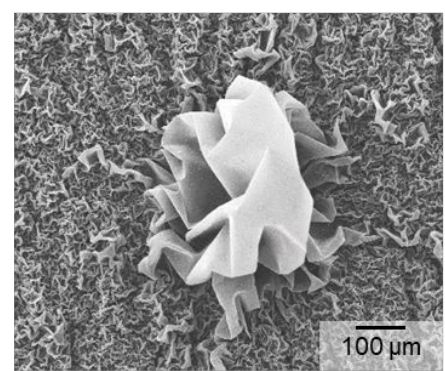

Convex Structure (b)

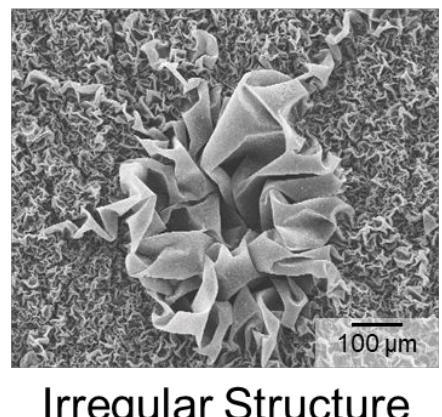

Irregular Structure (c)

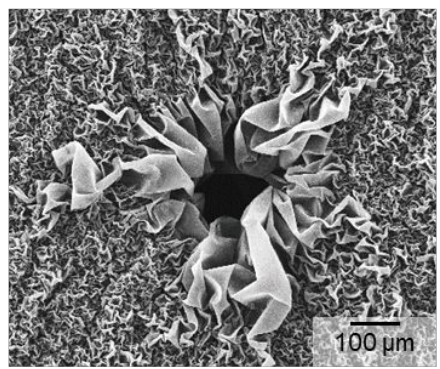

Concave Structure

Figure S5. Planar s-MXene films were deformed into (a) convex, (b) irregular, and (c) concave microstructures after the thermal shrinkage of a thermally-responsive PS substrate with an open hole. 


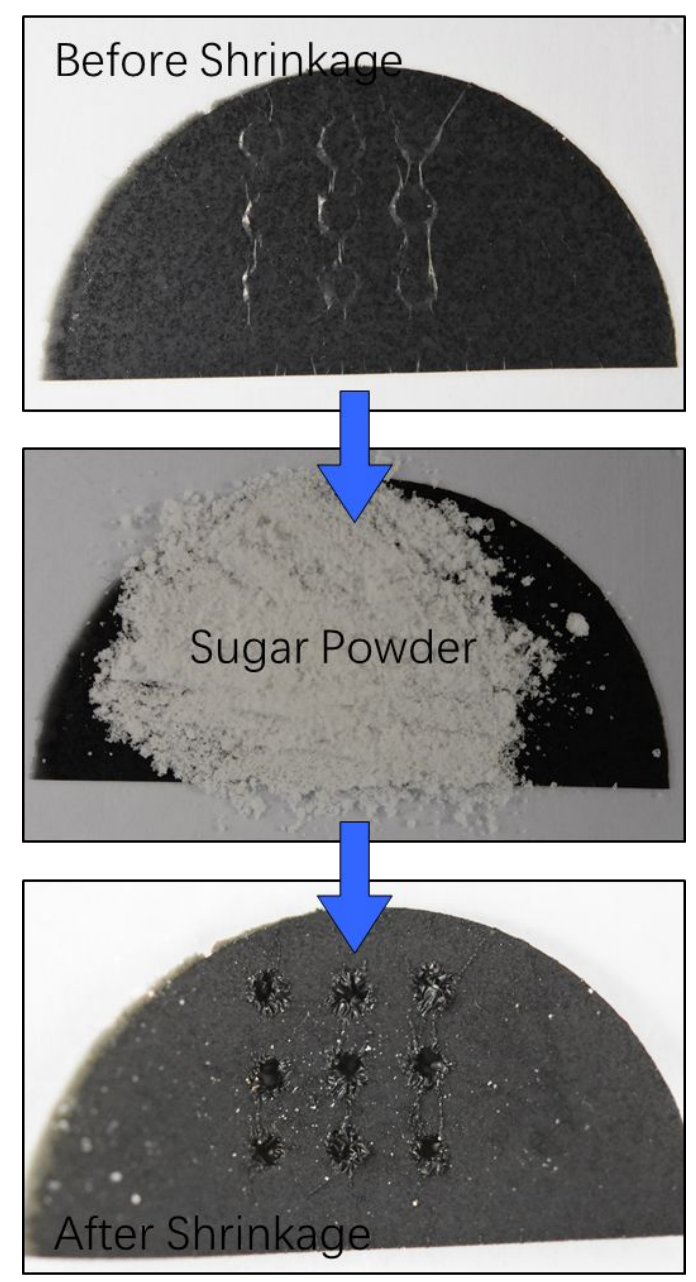

Figure S6. An array of s-MXene concave microstructures was specifically fabricated by placing sugar powders on the suspended s-MXene films prior to the thermal deformations/contractions. The s-MXene-coated samples were sandwiched between two steel plates to provide the normal forces to buckle the suspended s-MXene films downward. 

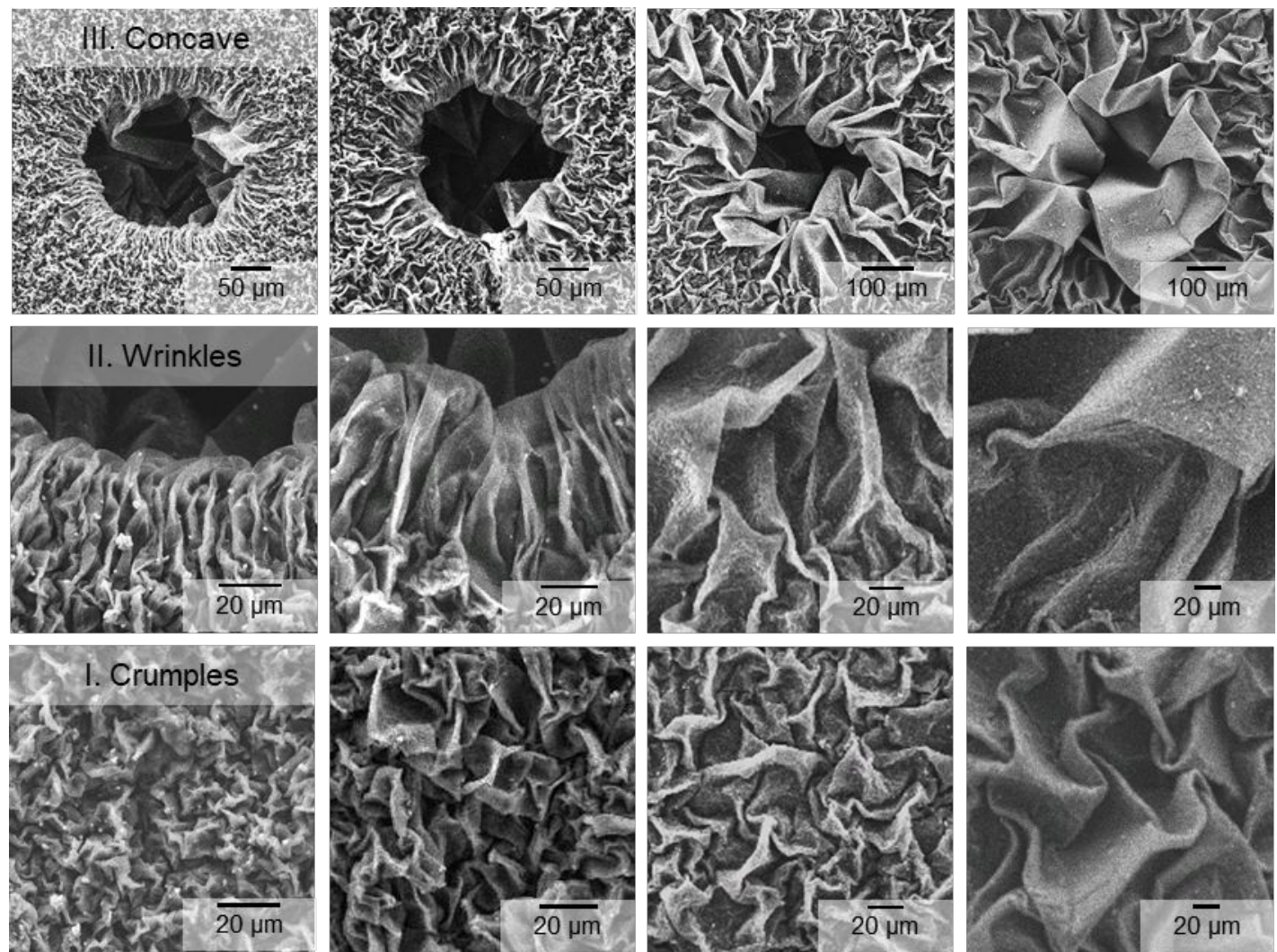

$0.7 \mu \mathrm{m}$

$1.1 \mu \mathrm{m}$

$1.9 \mu \mathrm{m}$

$2.7 \mu \mathrm{m}$

Increasing s-MXene Nanocoating Thickness

Figure S7. SEM images of heterogeneous s-MXene textures with tunable localized features by adjusting the thicknesses of s-MXene nanocoatings. 


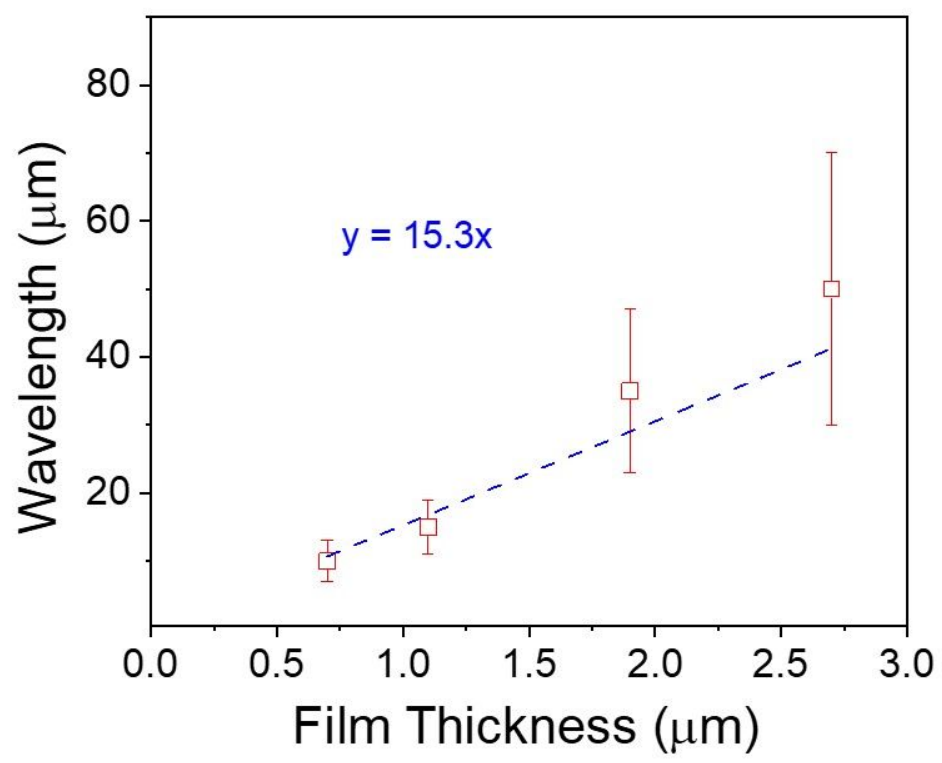

Figure S8. The dependency of wrinkling wavelength on the thickness of s-MXene nanocoatings. 
(a)
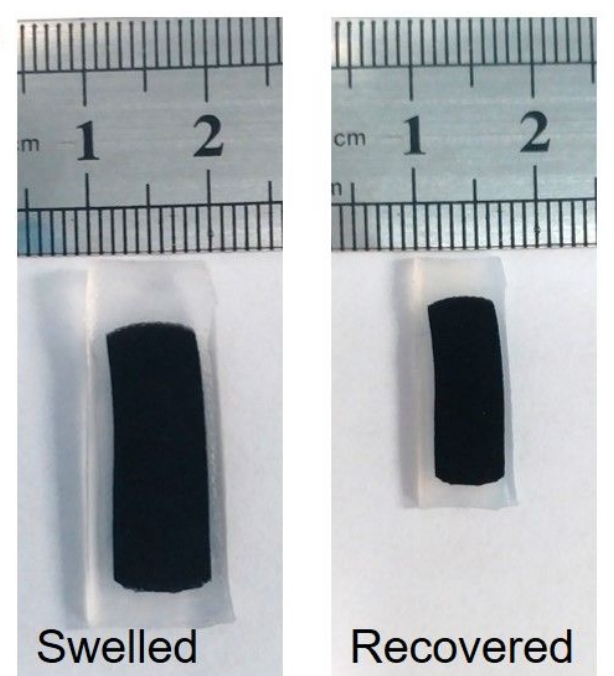

(b)
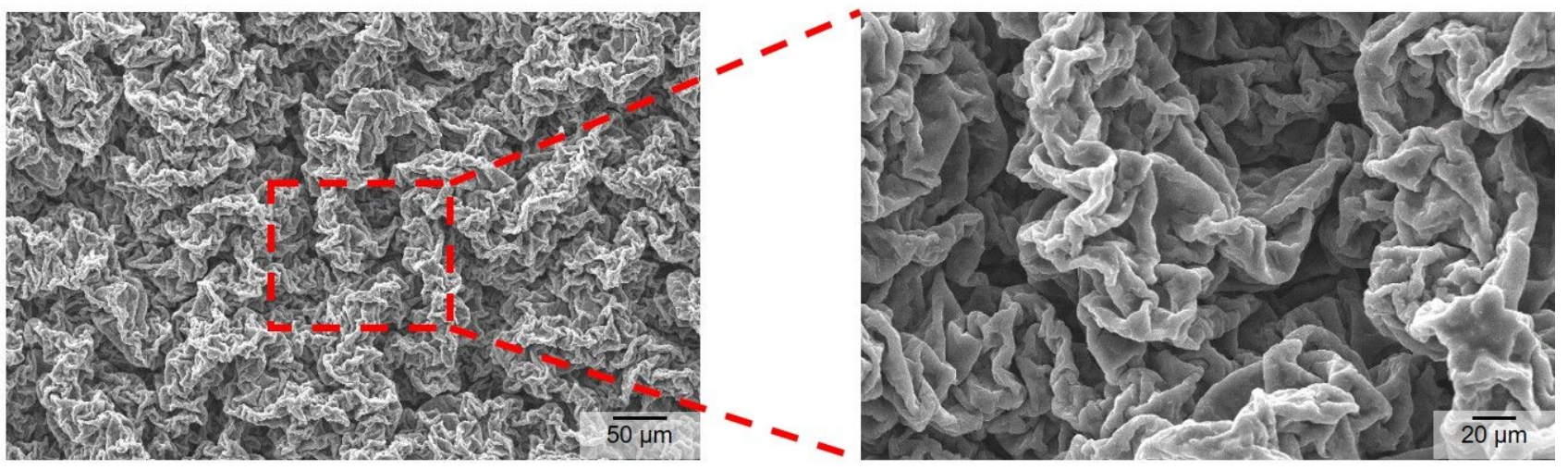

Figure S9. Transferring heterogeneous s-MXene textures from PS substrate to Ecoflex elastomer. (a) s-MXene/elastomer bilayer device under swelling and after complete DCM evaporation. (b) SEM images of isotropic s-MXene crumples on elastomeric substrate (after the transfer process). 
(a)

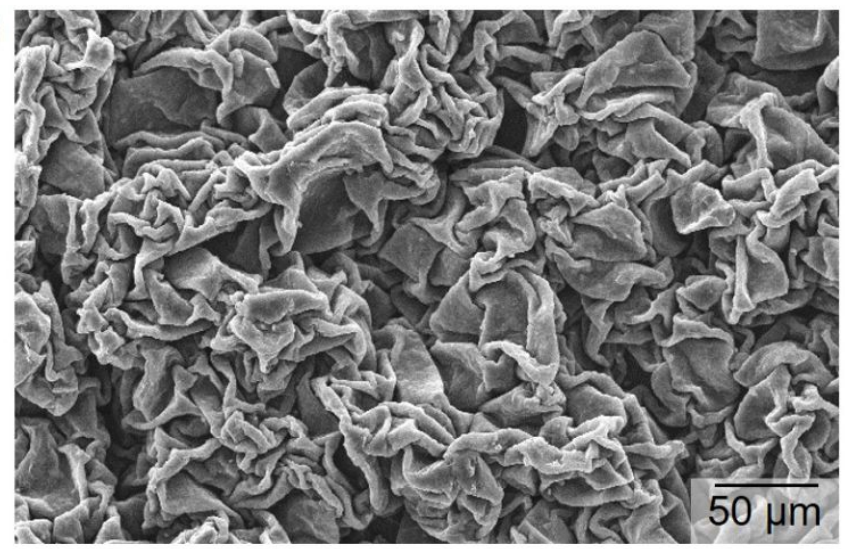

s-MXene on PS Substrate

(Before Transfer) (b)

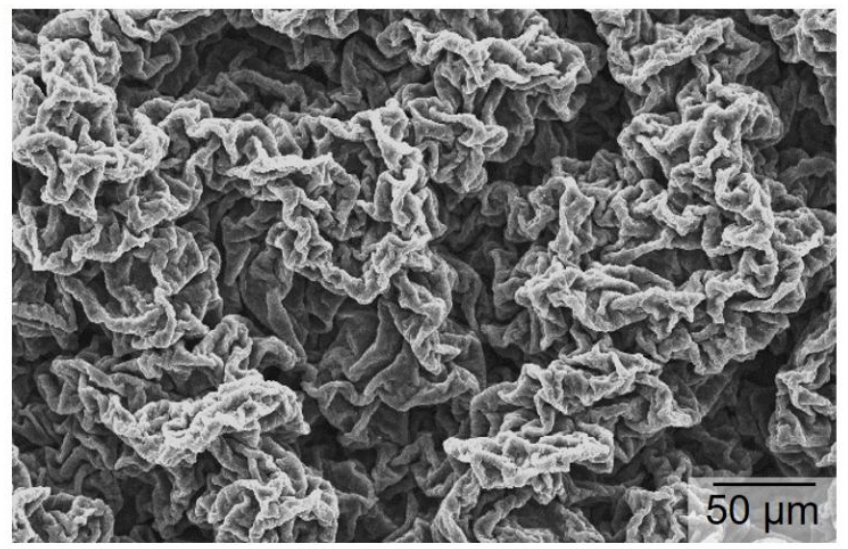

s-MXene on Elastomer Substrate (After Transfer)

Figure S10. (a) SEM image of isotropic s-MXene crumples on PS substrate (before the transfer process). (b) SEM image of isotropic s-MXene crumples on elastomeric substrate (after the transfer process).

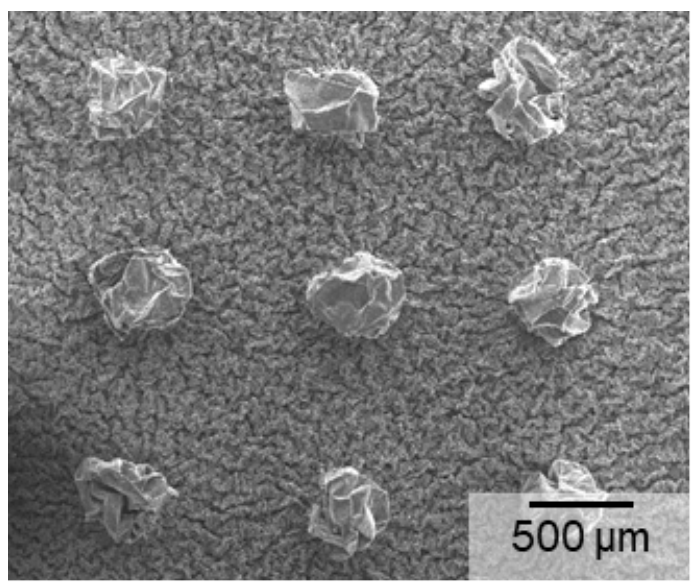

Figure S11. SEM image of a $3 \times 3$ array of s-MXene papillae after a 200-time tapping test (ca. $2 \mathrm{kPa})$. 


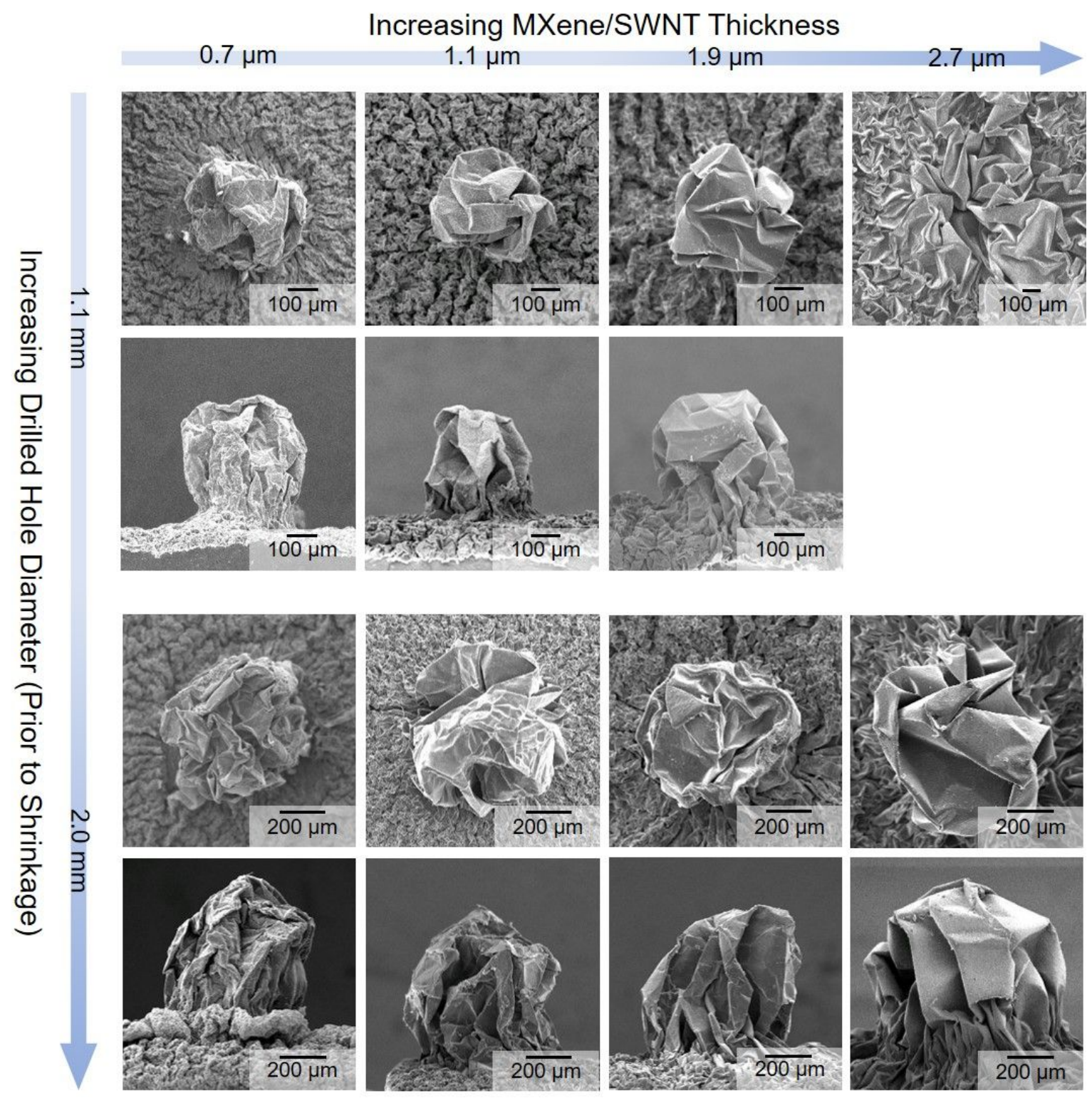

Figure S12. Library of SEM images of s-MXene papillae fabricated by using the s-MXene films with different thicknesses $(0.7$ to $2.7 \mu \mathrm{m})$ and the PS substrate with different hole diameters (1.1 and $2.0 \mathrm{~mm}$ ). 
(a)
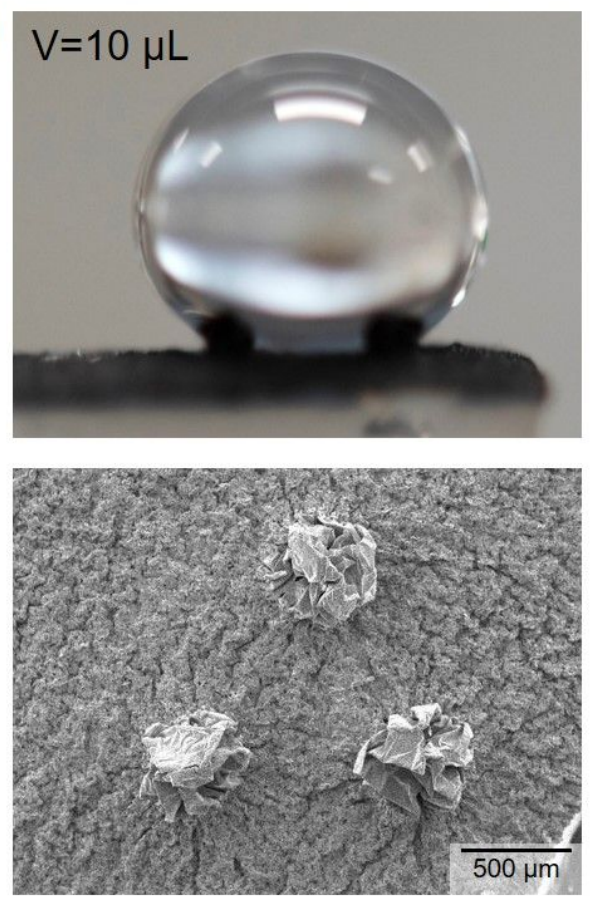

(b)
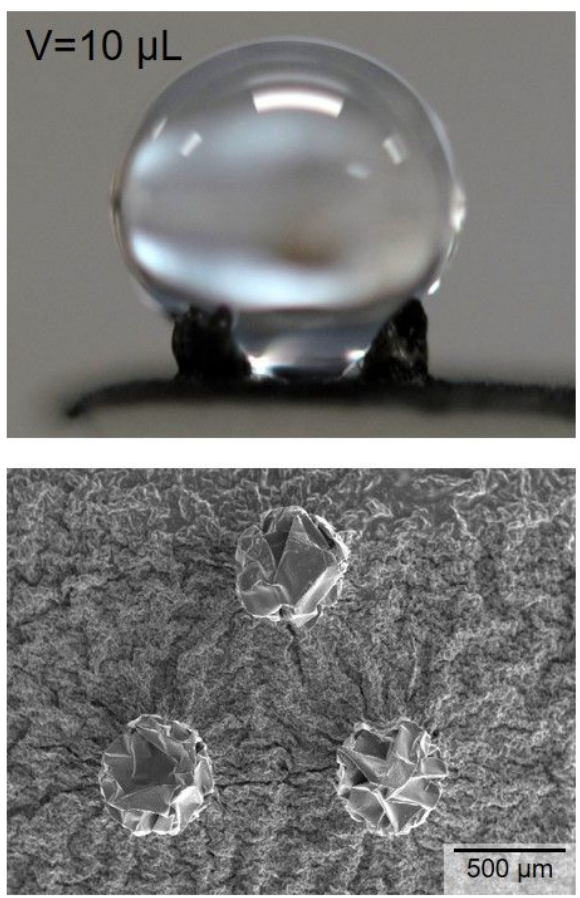

Figure S13. Microdroplet manipulation of triangle s-MXene papillae arrays with smaller diameters of (a) $430 \mu \mathrm{m}$ and (b) $530 \mu \mathrm{m}$. The two papillae arrays were not able to hold $10-\mu \mathrm{L}$ water droplets, and the water droplets contacted with the crumpled textures. 
(a)

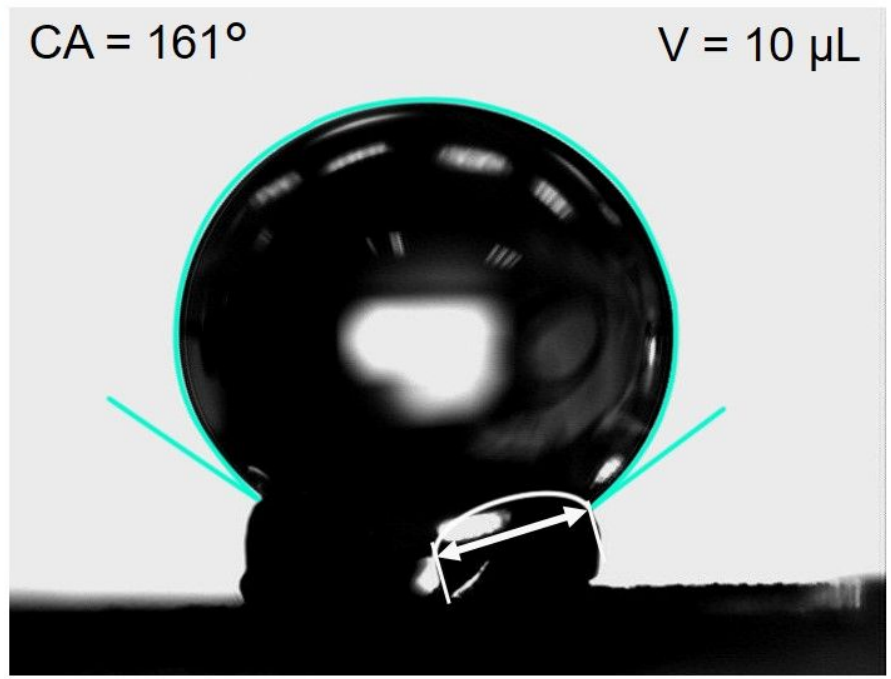

(b) $\mathrm{CA}=170^{\circ}$

$$
V=28 \mu \mathrm{L}
$$

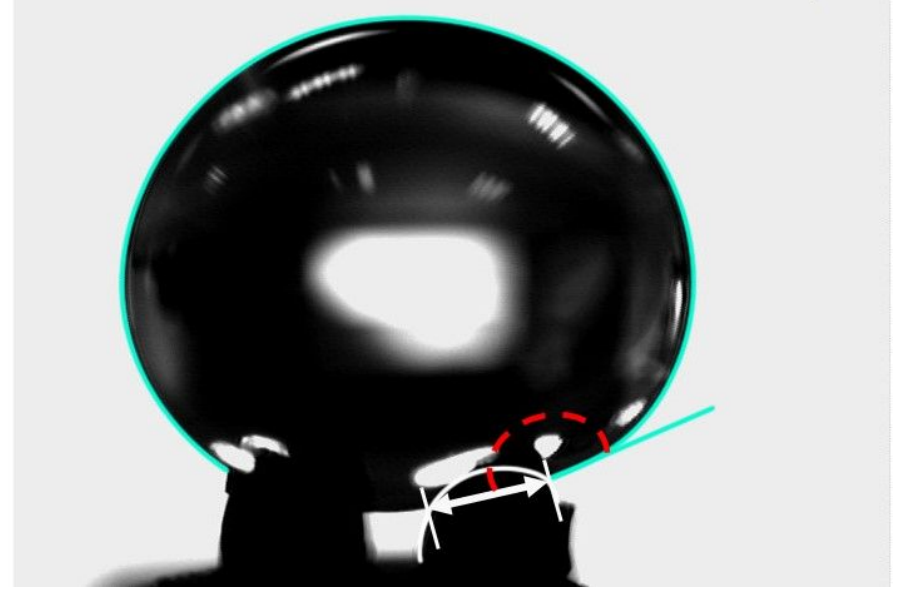

Figure S14. Measurement of water contact angle on (a) triangle papillae array and (b) square papillae array. 
(a)
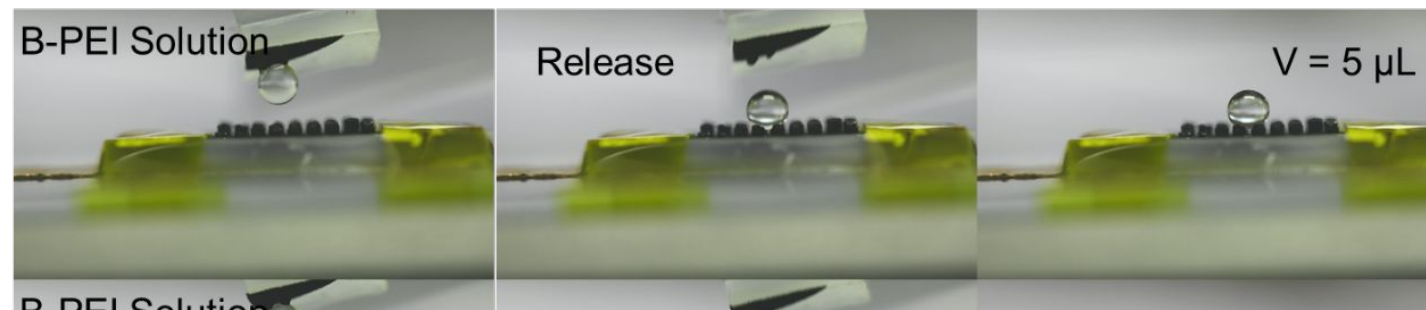

(b)
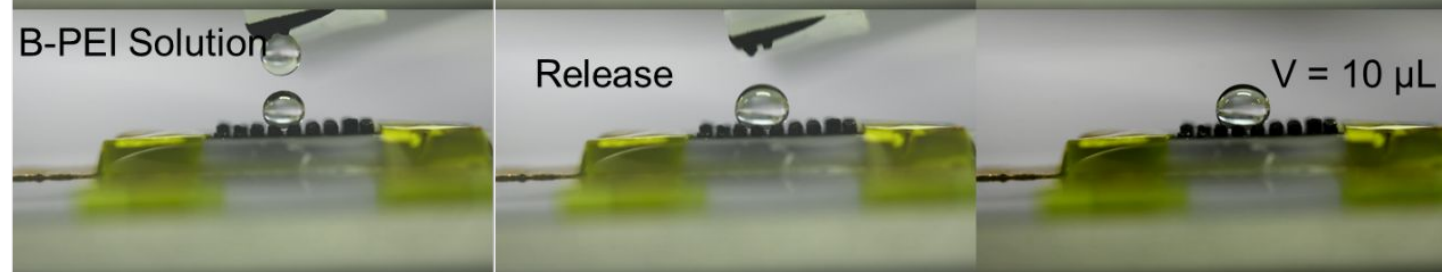

(c) Bacterial Solution

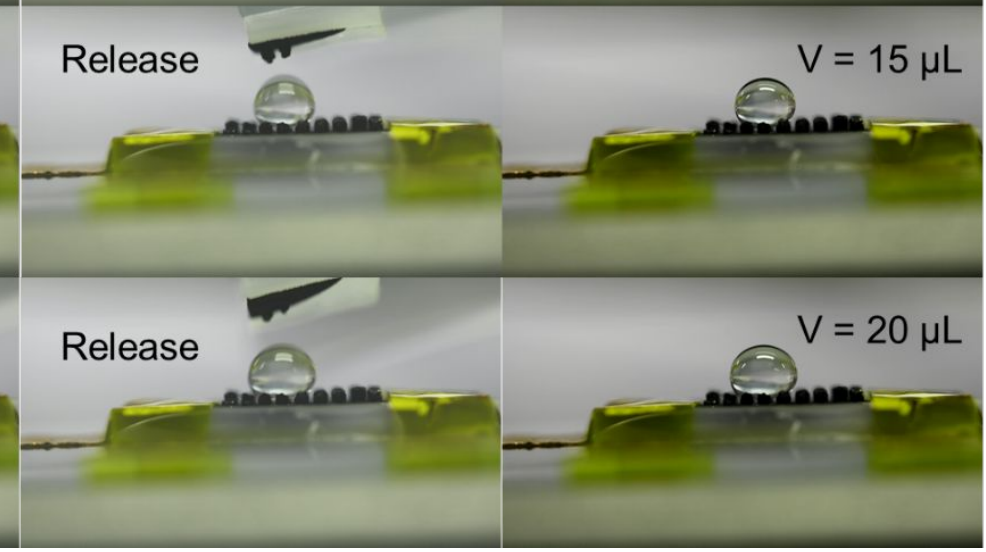

(d) Bacterial Solution

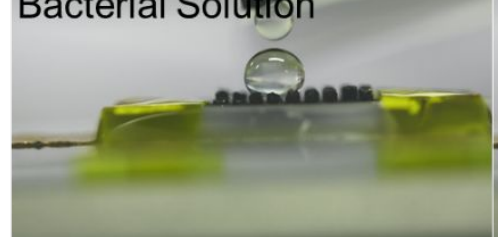

Figure S15. (a)(b) $10 \mu \mathrm{L}$ of B-PEI solution was transferred twice ( $5 \mu \mathrm{L}$ each time) from an sMXene manipulator with square papillae onto an array of s-MXene papillae under Joule heating. (c)(d) $10 \mu \mathrm{L}$ of bacterial solution was further transferred twice ( $5 \mu \mathrm{L}$ each time) from an s-MXene manipulator with square papillae to the stabilized B-PEI droplet. 


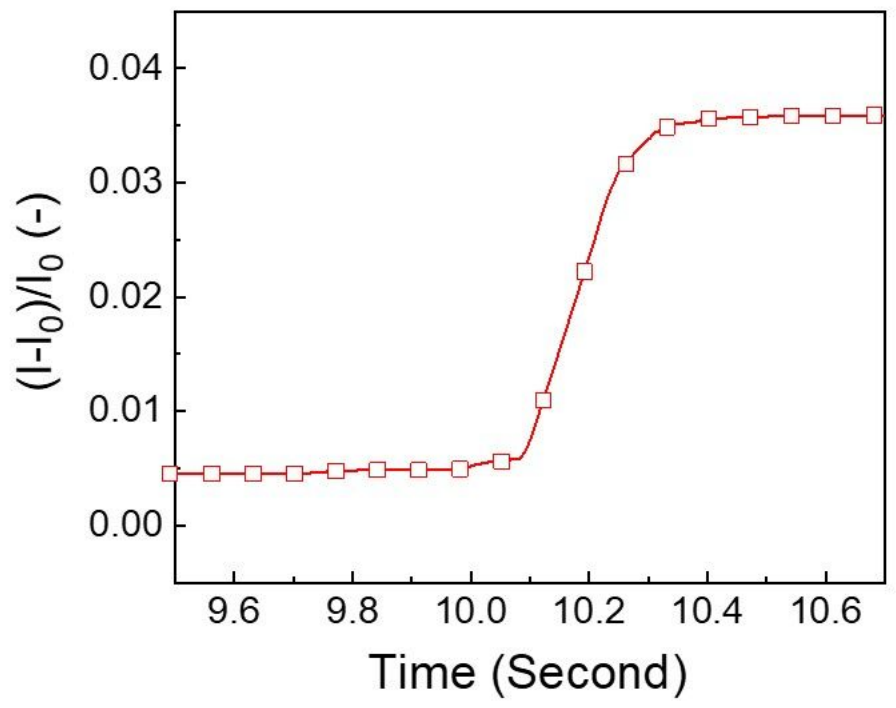

Figure S16. Relative current changes, $\left(I-I_{0}\right) / I_{0}$, of an s-MXene pressure sensor in response to a 13-Pa loading.

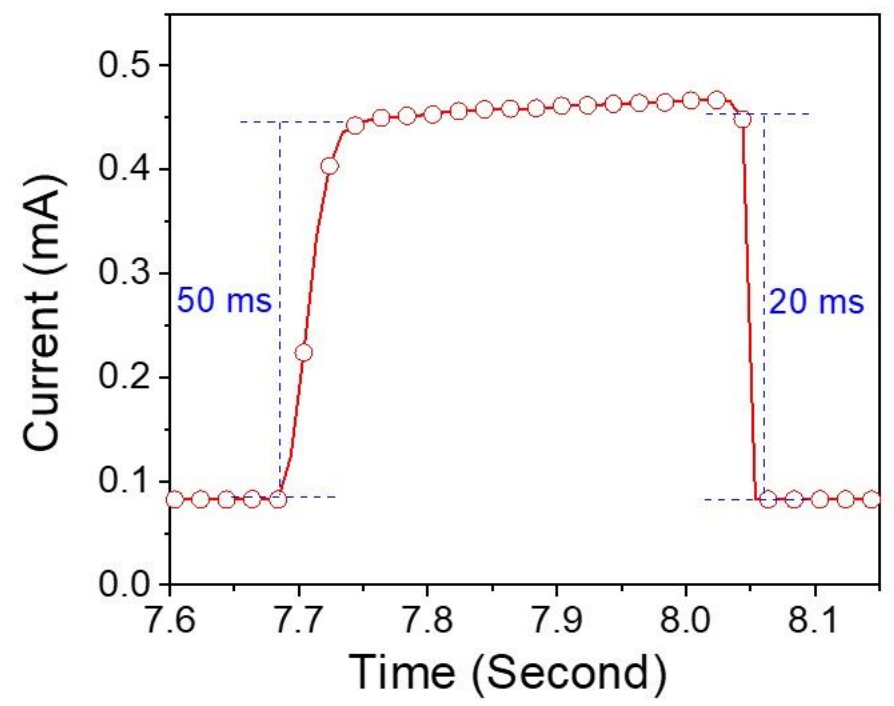

Figure S17. Measurement of response and recovery times of an s-MXene pressure sensor. 


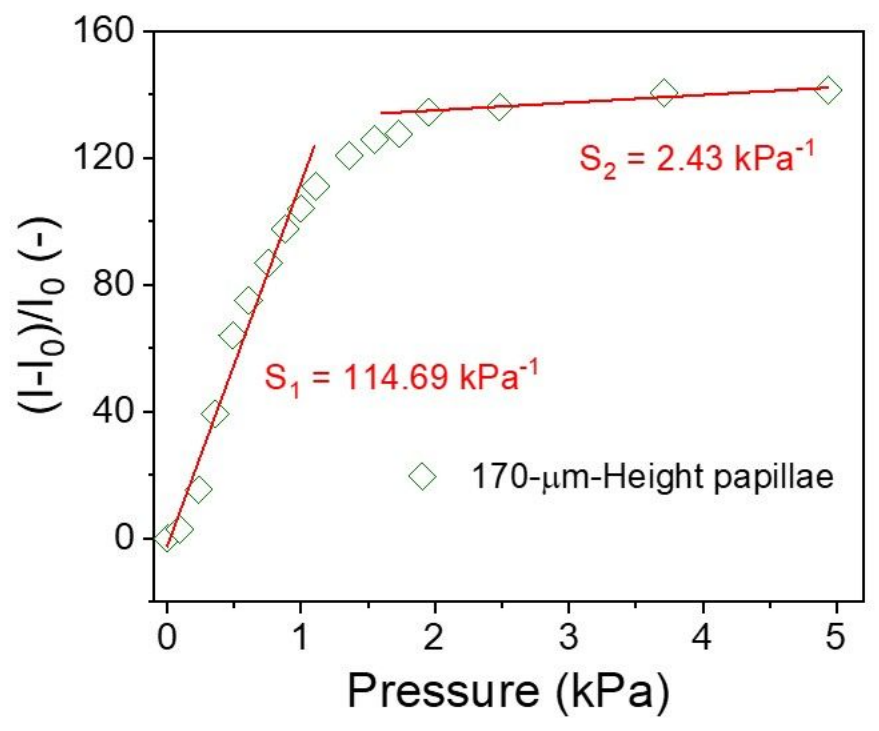

Figure S18. Relative current changes, $\left(I-I_{0}\right) / I_{0}$, of an s-MXene pressure sensor with the height of papillae at ca. $170 \mu \mathrm{m}$ with respect to various pressure loadings, where $I_{0}$ and $I$ are the measured currents at the unloaded and loaded states, respectively. 


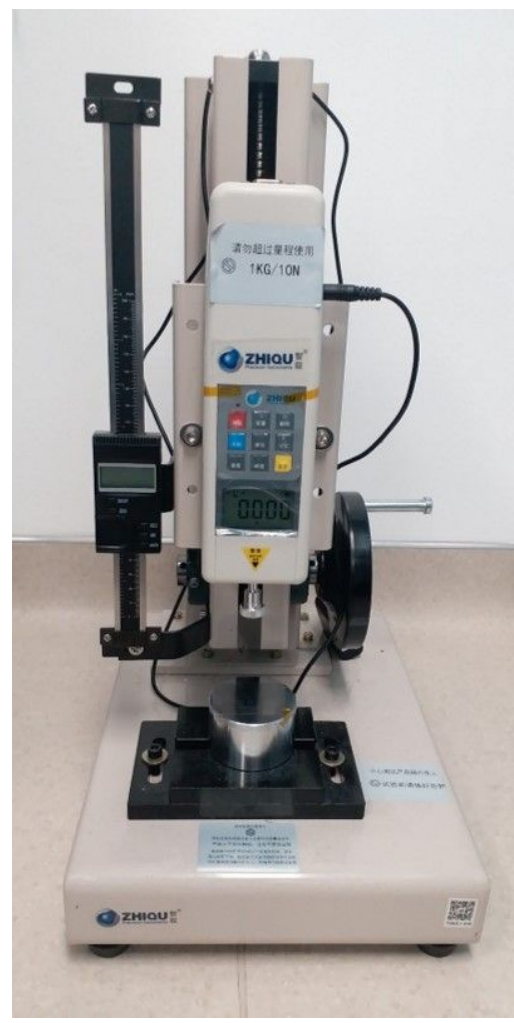

Figure S19. Homemade micro-stage system with a force gauge to apply normal force/pressure on s-MXene sensors. 
Table S1. Summary of reported MXene-based pressure sensors.

\begin{tabular}{|c|c|c|c|c|c|c|c|}
\hline \multirow[t]{2}{*}{ Sensing Materials } & \multicolumn{2}{|c|}{$\begin{array}{c}\text { Sensitivity } \\
\left(\mathrm{kPa}^{-1}\right)\end{array}$} & \multirow[t]{2}{*}{$\begin{array}{c}\text { Sensing Range } \\
\text { (Pa) }\end{array}$} & \multirow[t]{2}{*}{$\begin{array}{c}\text { Detection Limit } \\
\text { (Pa) }\end{array}$} & \multirow[t]{2}{*}{$\begin{array}{l}\text { Response Time } \\
\text { (ms) }\end{array}$} & \multirow[t]{2}{*}{$\begin{array}{c}\text { Cycling Stability } \\
(-)\end{array}$} & \multirow[t]{2}{*}{ Ref. } \\
\hline & $\left(\mathrm{I}-\mathrm{I}_{0}\right) /\left(\mathrm{I}_{0} \Delta \mathrm{P}\right)$ & $\left(\mathrm{R}-\mathrm{R}_{0}\right) /\left(\mathrm{R}_{0} \Delta \mathrm{P}\right)$ & & & & & \\
\hline \multirow{3}{*}{$\begin{array}{c}\text { MXene/ } \\
\text { Tissue Paper }\end{array}$} & $\begin{array}{l}0.55 \mathrm{kPa}^{-1} \\
(23-982 \mathrm{~Pa})\end{array}$ & \multirow{3}{*}{$-1-$} & \multirow{3}{*}{$23 \mathrm{~Pa}-30 \mathrm{kPa}$} & \multirow{3}{*}{10.2} & \multirow{3}{*}{11} & \multirow{3}{*}{10,000} & \multirow{3}{*}{39} \\
\hline & $\begin{array}{c}3.81 \mathrm{kPa}^{-1} \\
(0.982-10 \mathrm{kPa})\end{array}$ & & & & & & \\
\hline & $\begin{array}{c}2.52 \mathrm{kPa}^{-1} \\
(10-30 \mathrm{kPa})\end{array}$ & & & & & & \\
\hline \multirow{3}{*}{$\begin{array}{c}\text { MXene/Polyurethan } \\
\text { e/Chitosan }\end{array}$} & \multirow{3}{*}{$-1-$} & $\begin{array}{l}0.014 \mathrm{kPa}^{-1} \\
(<6.5 \mathrm{kPa})\end{array}$ & \multirow{3}{*}{$<240 \mathrm{kPa}$} & \multirow{3}{*}{9} & \multirow{3}{*}{$\sim 19$} & \multirow{3}{*}{5,000} & \multirow{3}{*}{40} \\
\hline & & $\begin{array}{c}-0.015 \mathrm{kPa}^{-1} \\
(6.5-85.1 \mathrm{kPa})\end{array}$ & & & & & \\
\hline & & $\begin{array}{l}-1.1 \times 10^{-3} \mathrm{kPa}^{-1} \\
(85.1-240 \mathrm{kPa})\end{array}$ & & & & & \\
\hline \multirow{2}{*}{$\begin{array}{c}\text { MXene Sponge/ } \\
\text { Polyvinyl Alcohol } \\
\text { Nanowires }\end{array}$} & $\begin{array}{c}147 \mathrm{kPa}^{-1} \\
(<5.37 \mathrm{kPa})\end{array}$ & \multirow{2}{*}{$-1-$} & \multirow{2}{*}{$<18.56 \mathrm{kPa}$} & \multirow{2}{*}{9} & \multirow{2}{*}{138} & \multirow{2}{*}{10,000} & \multirow{2}{*}{41} \\
\hline & $\begin{array}{c}442 \mathrm{kPa}^{-1} \\
(5.37-18.56 \mathrm{kPa})\end{array}$ & & & & & & \\
\hline \multirow{2}{*}{$\begin{array}{c}\text { MXene/ } \\
\text { Natural } \\
\text { Microcapsule }\end{array}$} & $\begin{array}{l}24.63 \mathrm{kPa}^{-1} \\
(10-200 \mathrm{~Pa})\end{array}$ & \multirow{2}{*}{$-/-$} & \multirow{2}{*}{$10 \mathrm{~Pa}-7 \mathrm{kPa}$} & \multirow{2}{*}{8} & \multirow{2}{*}{14} & \multirow{2}{*}{5,000} & \multirow{2}{*}{42} \\
\hline & $\begin{array}{l}1.18 \mathrm{kPa}^{-1} \\
(0.2-7 \mathrm{kPa})\end{array}$ & & & & & & \\
\hline \multirow{2}{*}{$\begin{array}{l}\text { MXene/Reduced } \\
\text { Graphene Oxide }\end{array}$} & $\begin{array}{l}4.05 \mathrm{kPa}^{-1} \\
(<1 \mathrm{kPa})\end{array}$ & & & & & & \\
\hline & $\begin{array}{l}22.56 \mathrm{kPa}^{-1} \\
(1-3.45 \mathrm{kPa})\end{array}$ & $-1-$ & $<3.45 \mathrm{kPa}$ & 10 & $<200$ & 10,000 & 43 \\
\hline MYom Toutil & $\begin{array}{l}3.844 \mathrm{kPa}^{-1} \\
(<29 \mathrm{kPa})\end{array}$ & 1 & $-101-\mathrm{D}$ & & 26 & & \\
\hline Mxene/ I ext1le & $\begin{array}{l}12.095 \mathrm{kPa}^{-1} \\
(29-40 \mathrm{kPa})\end{array}$ & $-1-$ & $<40 \mathrm{kPa}$ & - & 26 & 5,600 & 44 \\
\hline MXene & $-1-$ & $-1-$ & $0.35-12.08 \mathrm{kPa}$ & - & $<30$ & 4,000 & 6 \\
\hline MXene/Carbon & $\begin{array}{l}11.47 \mathrm{kPa}^{-1} \\
(13-770 \mathrm{~Pa})\end{array}$ & $\begin{array}{l}-0.87 \mathrm{kPa}^{-1} \\
(13-770 \mathrm{~Pa})\end{array}$ & 10 & & & 10000 & This \\
\hline Nanotube/Ecoflex & $\begin{array}{l}0.23 \mathrm{kPa}^{-1} \\
(2-10 \mathrm{kPa})\end{array}$ & $\begin{array}{l}-0.002 \mathrm{kPa}^{-1} \\
(2-10 \mathrm{kPa})\end{array}$ & $13 \mathrm{~Pa}-10 \mathrm{kPa}$ & 13 & 50 & 10,000 & work \\
\hline
\end{tabular}

